\title{
OPEN Timeliness of antiretroviral therapy initiation in the era before universal treatment
}

\begin{abstract}
Nikolina Bogdanić ${ }^{1}$, Liam Bendig ${ }^{2}$, Davorka Lukas $^{1,3}$, Šime Zekan $^{1,3}$ \& Josip Begovac ${ }^{1,3 凶}$
We assessed the prevalence and factors related to the time to antiretroviral (ART) initiation among persons who entered HIV care and subsequently started ART in Croatia from 2005 to 2014. Included were patients $\geq 18$ years, the follow-up ended on Dec/31/2017. 628 patients were included into the study 91.9\% were men; median age was 36.1 (01-03: 29.6-43.8) years. Rapid (within 7 days of diagnosis) ART initiation was observed in $21.8 \%$ patients, $49.8 \%$ initiated ART within 30 days, $21.7 \%$ and $28.5 \%$ had intermediate ( 31 days -1 year) and late initiation ( $>1$ year), respectively. Of 608 patients that achieved an undetectable viral load, $94 \%$ had a plasma HIV-1 RNA $<50$ copies/ml at last measurement after a median follow-up of 5.2 years. On quantile regression analysis, calendar year of entry into care, and markers of more advanced HIV disease (higher viral load, lower CD4 cell count and clinical AIDS) were significantly associated with earlier ART initiation. Early ART was not related to a gap in care afterwards at all quantiles. In conclusion, a significant proportion of patients started ART early in Croatia in 2005-2014. Early ART initiation led to durable viral load suppression and was not associated with a subsequent gap in care.
\end{abstract}

Guidelines concerning antiretroviral therapy (ART) initiation have evolved over the past decade. While there was a consensus that ART should be started as soon as possible in patients with clinical AIDS, a CD4 cell count threshold for ART initiation has been debated since the introduction of modern ART in 1996. In 2010 WHO recommended ART for patients with a CD4 cell count beneath 350 cells $/ \mu \mathrm{L}^{1}$. The landmark START trial ${ }^{2}$ in conjunction with other randomized trials ${ }^{3}$ and observational studies ${ }^{4,5}$ led to a policy change in 2015 by recommending ART for all patients regardless of the CD4 cell count ${ }^{6}$. Another WHO update in 2017 recommended rapid initiation (within 7 days of diagnosis) of ART to indicated, willing patients ${ }^{7}$. These guidelines also raised concerns over the elimination of the series of standard counseling sessions preceding ART due to a possibility of resulting physical or psychological harm and potential non-adherence ${ }^{7}$. The 2020 European AIDS Clinical Society guidelines also mention the importance of rapid ART initiation but emphasizes patient understanding and readiness to begin, as do current U.S. Department of Health and Human Services recommendations ${ }^{8,9}$.

Several low- and middle-income countries have introduced rapid ART initiation as a means to address the challenge of follow-up loss and late patient presentation to care ${ }^{10}$. This policy is linked to faster viral suppression for people living with HIV (PLWH) which also means that the risk of onward transmission can be greatly reduced $^{10}$. Another consideration in rapid ART is the feasibility of implementation. Retrospective evaluation of rapid-start ART in community settings at risk for loss to follow-up, comorbidities, and other vulnerabilities show that implementation is possible and successful in these instances ${ }^{11,12}$. Even in the setting of high HIV prevalence communities experiencing a variety of health care challenges, the goal of reducing time to ART initiation from diagnosis is achievable ${ }^{11}$. However, a recent study from the French Dat'AIDS cohort examining patients diagnosed with HIV between 2010 and 2015 reported that a longer time between first encounter and first ART prescription was associated with a better 1 -year retention and higher viral load suppression at 6 months ${ }^{13}$.

In the era before universal ART, data on the timing of ART initiation from a patient's documented HIV infection or first clinical visit was generally not highlighted and seldom reported. The aim of our study was to examine the timeliness of ART initiation among persons who entered HIV care in Croatia from 2005 to 2014. We sought to identify factors associated with differences in timing of ART.

${ }^{1}$ University Hospital for Infectious Diseases, Mirogojska 8, Zagreb, Croatia. ${ }^{2}$ Medical Scholars Program, AU/UGA Medical Partnership, Athens, GA, USA. ${ }^{3}$ University of Zagreb School of Medicine, Zagreb, Croatia. ${ }^{\square}$ email: josip.begovac@gmail.com 


\section{Results}

A total of 628 patients were included into the study with a total of 4333.0 years of follow-up, of which 3684.9 (85\%) years of follow-up were after ART initiation. The median follow-up time per person was 6.7 (Q1-Q3: 4.3-9.5) years and the median time on ART was 5.4 (Q1-Q3: 3.5-8.2) years. The main characteristics of our study population are presented in Table 1. There was a trend for earlier initiation of ART among older patients, those with more severe HIV-disease (lower CD4 cell count, clinical AIDS, higher viral load). Earlier ART initiation was observed more frequently in the more recent period (2012 to 2014) and among those living outside Zagreb as well as among persons with heterosexual mode of transmission (Tables 1 and 2). Among 244 patients with a CD4 cell count $\geq 350 / \mathrm{mm}^{3}$ at inclusion into care ART initiation within 30 days of HIV diagnosis was observed in $40(16.4 \%)$, it was more frequent in the period 2012 to $2014(30 / 101,29.7 \%)$ compared to the period 2005 to $2011(10 / 143,7.0 \%)$. Median time from confirmed HIV test to first clinical visit was 7 (Q1-Q3: 2-15) days; this integration into care was within 7 days in 339 (54.0\%) and within 1-month in 540 (86.0\%) patients.

Timeliness of ART. The median time from HIV diagnosis to ART initiation was 31 days (Q1-Q3: 8-537.5 days; $0.3-17.7$ months), decreasing by $77.8 \%$ from the period 2005-2008 to 2012-2014 (Table 2). The proportion of patients with early ART (<30 days of confirmed diagnosis) was significantly greater in the period of 2012-2014 than from 2005 to 2008 (Fig. 1a). About 20\% of patients started ART within 7 days of HIV-diagnosis, but there was no statistically significant difference in different study periods (Fig. 1a, $\mathrm{P}=0.618$ ). The proportion of patients entering care from 2005 to 2008 also had significantly greater delay in ART initiation of more than one, two or three years after diagnosis (Fig. 1a).

In patients entering care between 2005 and 2008, the median time from the first clinical visit to ART initiation was 1.3 months (41 days) (Q1-Q3: 0.1-34.8 months; 4-1058 days). Patients from 2009 to 2011 had a median time from HIV diagnosis to ART initiation of 1.0 months (Q1-Q3: 0.3-16.9 months) and 0.5 months (14.5 days) from first clinical visit to ART initiation (Q1-Q3: 0.0-13.6 months; 1-412.5 days). Patients in the most recent studied time period, 2012-2014, had the earliest initiation of ART, the median time from diagnosis to ART initiation was 0.6 months (18 days) (Q1-Q3: 0.3-6.2 months; 8-188 days) and 0.2 months (6 days) (Q1-Q3: 0.0-3.3 months; 1-99.5 days) from the first clinical visit.

The proportion of patients starting ART from the date of first clinical visit follows the pattern of the time from HIV diagnosis to ART initiation (Fig. 1b). Median times to ART initiation and 95\% CI for different patients' characteristics are presented in Table 2.

Viral suppression. We estimated the time from HIV diagnosis to reaching undetectable $(<50$ copies $/ \mathrm{mL})$ HIV-1 RNA by the Kaplan-Meier product-limit survival estimates. Overall, of 628 patients 608 (96.8\%) became undetectable during follow-up. Of the 20 who did not, 15 died and 5 were lost to follow-up (LTFU). The median time to reach an undetectable viral load (VL) was 9.6 months (95\% CI 7.9-10.6) for the whole study population (Fig. 2a). The median number of months for reaching an undetectable VL decreased by $27.7 \%$ from the period 2005-2008 compared to the period 2012-2014 ( $<$ 0.001) (Table 2). Patients who had a gap in care had a longer time to virologic suppression (median 38.2, 95\% CI: 26.9-49.9 months) compared to those who did not have a gap (median 7.5, 95\% CI 6.8-8.7 months; $\mathrm{P}<0.001$ ) (Fig. 2b) (Table 2). Those with a gap in care before ART initiation were suppressed after a median of 67.0 (95\% CI 58.9-79.3) months after HIV diagnosis; those who had a gap after ART initiation were suppressed after a median 11.4 (95\% CI 6.7-14.1) months. Other significant associations with viral suppression were related to the study period (Fig.3c), timing of ART initiation (Fig. 3d) and the severity of HIV disease (lower CD4 cell count, higher viral load and clinical AIDS) (Table 2).

Virological failure, defined as one or more plasma HIV-1 RNA measurement $>1000$ copies $/ \mathrm{ml}$, was observed in 92 (15.3\%) of 601 patients who had a viral load test done 6-months after ART initiation (Table 3). Of 51 persons in whom at least one HIV-1 resistance test was done, resistance was detected in 28 (54.9\%). Of 131 rapid ART initiators 12 (9.2\%) had one or more clinically relevant resistance mutations and this proportion was higher than in the early $(4 / 168,2.4 \%)$, intermediate $(5 / 131,3.8 \%)$ or late $(7 / 171,4.1 \%)$ ART initiation groups. The majority of persons with virological failures were resuppressed to $<50$ copies $/ \mathrm{ml}$ at last HIV-1 RNA measurement (Table 3).

Of 608 patients who reached an undetectable viral load $573(94.2 \%)$ had $<50$ copies of HIV-1 RNA per ml at the last viral load measurement (median: 5.2, Q1-Q3: 3.3-8.0 years of follow-up). Of the whole study population $(\mathrm{N}=628), 574(91.4 \%)$ had $<50$ copies of HIV-1 RNA per $\mathrm{ml}$ at the last viral load measurement after a median of 5.1 (Q1-Q3: 3.2-7.9) years of follow-up.

Gap in care. In our cohort, 120 patients (19.1\%) experienced at least 1 gap of $>12$ months in care, $28(4.5 \%)$ had $\geq 2$ gaps and 9 (1.4\%) had $\geq 3$ gaps in care over their follow-up period. After the first gap $85.0 \%(102 / 120)$ patient had a subsequent visit. After the last gap, altogether 26 of $120(21.7 \%)$ patients had no subsequent visit within 12 months till the end of follow-up and were considered LTFU. Of the 120 persons with a gap in care, 62 (51.7\%) had a gap after and $49(40.8 \%)$ before ART start; nine (7.5\%) had both a gap before and after ART start.

There was a total of 159 episodes of gaps with a median length of 579 days (Q1-Q3: 415.0-1099.0 days). There were 76 episodes (11.7 per 100 patient years of follow-up) of gap before ART initiation and 83 (2.2 per 100 patient years of follow-up) after ART initiation. The rate ratio suggests that a gap before ART initiation was 5.2 times more likely than a gap after ART initiation (95\% CI 3.8-7.1).

Multivariable analysis. We examined factors related to the time from confirmed HIV diagnosis to ART initiation by quantile regression analysis (Fig. 3). A gap in care before ART initiation, calendar year of entry into care, and markers of more advanced HIV disease (higher viral load, lower CD4 cell count and clinical AIDS) were significantly associated with time of ART initiation at all quantiles. Gap after ART, gender, transmission 


\begin{tabular}{|c|c|c|c|c|c|c|}
\hline \multirow[b]{2}{*}{ Variables } & \multirow[b]{2}{*}{$\begin{array}{l}\text { Total } \\
N=628(\%)\end{array}$} & \multicolumn{4}{|c|}{ ART initiation after confirmed HIV diagnosis } & \multirow[b]{2}{*}{ P-value } \\
\hline & & $\begin{array}{l}\text { Rapid } \leq 7 \text { days } \quad \mathrm{N}=137 \\
(\%)\end{array}$ & $\begin{array}{l}\begin{array}{l}\text { Early 8-30 days } \mathrm{N}=176 \\
(\%)\end{array}\end{array}$ & $\begin{array}{l}\text { Intermediate } 31 \text { day-1 } \\
\text { year } \quad \mathrm{N}=136(\%)\end{array}$ & $\begin{array}{l}\text { Delayed }>1 \text { year } \mathrm{N}=179 \\
(\%)\end{array}$ & \\
\hline \multicolumn{7}{|l|}{ Baseline characteristics } \\
\hline Male sex & $577(91.9)$ & $122(89.1)$ & $163(92.6)$ & $126(92.6)$ & $166(92.7)$ & 0.297 \\
\hline Age, years & $36.1(29.6-43.8)$ & $39.3(32.8-48.0)$ & $38.1(30.9-46.8)$ & $34.3(27.8-43.1)$ & $32.7(28.2-39.1)$ & $<0.001$ \\
\hline Age groups & & & & & & $<.001$ \\
\hline$<30$ years & $169(26.9)$ & $21(15.3)$ & $39(22.2)$ & $44(32.4)$ & $65(36.3)$ & \\
\hline $30-50$ years & $374(59.6)$ & $90(65.7)$ & $103(58.5)$ & $77(56.6)$ & $104(58.1)$ & \\
\hline$>50$ years & $85(13.5)$ & $26(19.0)$ & $34(19.3)$ & $15(11.0)$ & $10(5.6)$ & \\
\hline Mode of transmission & & & & & & $0.050^{\mathrm{a}}$ \\
\hline MSM & $476(75.8)$ & $99(72.3)$ & $129(73.3)$ & $106(77.9)$ & $142(79.3)$ & \\
\hline Heterosexual contact & $121(19.3)$ & $31(22.6)$ & $40(22.7)$ & $22(16.2)$ & $28(15.6)$ & \\
\hline IDU & $11(1.8)$ & $2(1.5)$ & $1(0.6)$ & $4(2.9)$ & $4(2.2)$ & \\
\hline Unknown & $20(3.2)$ & $5(3.6)$ & $6(3.4)$ & $4(2.9)$ & $5(2.8)$ & \\
\hline $\begin{array}{l}\text { Periods of integration into } \\
\text { care, years }\end{array}$ & & & & & & $<0.001$ \\
\hline $2005-2008$ & $220(35.0)$ & $46(33.6)$ & $40(22.7)$ & $44(32.4)$ & $90(50.3)$ & \\
\hline $2009-2011$ & $176(28.0)$ & $38(27.7)$ & $51(29.0)$ & $37(27.2)$ & $50(27.9)$ & \\
\hline $2012-2014$ & $232(36.9)$ & $53(38.7)$ & $85(48.3)$ & $55(40.4)$ & $39(21.8)$ & \\
\hline Residency in Zagreb & $273(43.5)$ & $61(44.5)$ & $71(40.3)$ & $41(30.1)$ & $100(55.9)$ & 0.074 \\
\hline $\begin{array}{l}\text { CD } 4 \text { count at inclusion into } \\
\text { care, cells } / \mu \mathrm{L}\end{array}$ & $269.0(69.0-445.0)$ & $93.0(34.0-209.0)$ & $107.0(31.5-269.5)$ & $316.0(169.0-456.0)$ & $484.0(372.0-633.0)$ & $<0.001$ \\
\hline $\begin{array}{l}\text { CD4 count groups at inclu- } \\
\text { sion into care, cells } / \mu \mathrm{L}\end{array}$ & & & & & & $<.001$ \\
\hline$<100$ & $190(30.3)$ & $71(51.8)$ & $87(49.4)$ & $24(17.6)$ & $8(4.5)$ & \\
\hline $100-350$ & $194(30.9)$ & $50(36.5)$ & $65(36.9)$ & $53(39.0)$ & $26(14.5)$ & \\
\hline$\geq 350$ & $244(38.9)$ & $16(11.7)$ & $24(13.6)$ & 59 (43.4) & $145(81.0)$ & \\
\hline $\begin{array}{l}\text { Viral load at inclusion into } \\
\text { care, } \log 10\end{array}$ & $5.1(4.5-5.7)$ & $5.6(5.1-6.1)$ & $5.4(4.9-5.8)$ & $5.1(4.4-5.5)$ & $4.5(4.0-5.0)$ & $<.001$ \\
\hline $\begin{array}{l}\text { Viral load at inclusion into } \\
\text { care }>100000 \mathrm{c} / \mathrm{mL}\end{array}$ & $341(54.3)$ & $105(76.6)$ & $117(66.5)$ & $74(54.4)$ & $45(25.1)$ & $<.001$ \\
\hline $\begin{array}{l}\text { Hepatitis B antigen present } \\
\text { at inclusion into care }\end{array}$ & $34(5.4)$ & $5(3.6)$ & $11(6.3)$ & $7(5.1)$ & $11(6.1)$ & 0.462 \\
\hline $\begin{array}{l}\text { Hepatitis C antibody posi- } \\
\text { tive at inclusion into care }\end{array}$ & $19(3.0)$ & $1(0.7)$ & $6(3.4)$ & $6(4.4)$ & $6(3.4)$ & 0.197 \\
\hline $\begin{array}{l}\text { AIDS at inclusion into care } \\
\text { present }\end{array}$ & $186(29.6)$ & $49(35.8)$ & $81(46.0)$ & $29(21.3)$ & $27(15.1)$ & $<0.001$ \\
\hline \multicolumn{7}{|l|}{ Follow-up characteristics } \\
\hline $\begin{array}{l}\text { CD4 count at ART initia- } \\
\text { tion, cells } / \mu \mathrm{L}\end{array}$ & $225.5(62.0-355.5)$ & $93.0(34.0-209.0)$ & $107.0(31.5-273.0)$ & $265.5(154.5-401.0)$ & $330.0(229.0-454.0)$ & $<.001$ \\
\hline $\begin{array}{l}\text { CD4 count groups on ART, } \\
\text { cells } / \mu \mathrm{L}\end{array}$ & & & & & & $<0.001$ \\
\hline$<100$ & $211(33.6)$ & $71(51.8)$ & $87(49.4)$ & $25(18.4)$ & $28(15.6)$ & \\
\hline $100-350$ & $253(40.3)$ & $51(37.2)$ & $63(35.8)$ & $69(50.7)$ & $70(39.1)$ & \\
\hline$\geq 350$ & $164(26.1)$ & $15(10.9)$ & $26(14.8)$ & $42(30.9)$ & $81(45.3)$ & \\
\hline $\begin{array}{l}\text { Viral load at ART initiation, } \\
\log 10\end{array}$ & $5.2(4.6-5.7)$ & $5.6(5.1-6.1)$ & $5.4(4.9-5.8)$ & $5.1(4.5-5.5)$ & $4.6(4.3-5.3)$ & $<.001$ \\
\hline ART type & & & & & & $0.003^{\mathrm{b}}$ \\
\hline 2NRT + NNRTI & $424(67.5)$ & $77(56.2)$ & $124(70.5)$ & $99(72.8)$ & $124(69.3)$ & \\
\hline 2NRTI+PI & $170(27.1)$ & $55(40.1)$ & $42(23.9)$ & $34(25.0)$ & $39(21.8)$ & \\
\hline 2NRTI+II & $26(4.1)$ & $2(1.5)$ & $7(4.0)$ & $2(1.5)$ & $15(8.4)$ & \\
\hline Other & $8(1.3)$ & $3(2.2)$ & $3(1.7)$ & $1(0.7)$ & $1(0.6)$ & \\
\hline Had gap in care & $120(19.1)$ & $15(10.9)$ & $14(8.0)$ & $21(15.4)$ & $70(39.1)$ & $<0.001$ \\
\hline Died & $50(8.0)$ & $10(7.3)$ & $21(11.9)$ & $8(5.9)$ & $11(6.1)$ & 0.267 \\
\hline
\end{tabular}

Table 1. Main characteristics of the entire study cohort and of each group based on antiretroviral therapy initiation after confirmed HIV diagnosis. Values are frequencies, percentages and median with first and third quartiles (Q1, Q3). MSM men who have sex with men; IDU injecting drug use; $A R T$ antiretroviral therapy; NRTI nucleoside analogues reverse transcriptase inhibitors; NNRTI nonnucleoside reverse transcriptase inhibitors; PI protease inhibitors, $I I$ integrase inhibitors. ${ }^{a}$ Cohrane-Armitage trend test for comparison of MSM versus heterosexual mode of transmission. ${ }^{\mathrm{b}}$ Cohrane-Armitage trend test for the comparison of $2 \mathrm{NRTI}+\mathrm{NNRTI}$ versus 2NRTI + PI. 


\begin{tabular}{|c|c|c|c|}
\hline Variables & N (\%) & $\begin{array}{l}\text { Time from HIV diagnosis to ART initiation, } \\
\text { months }\end{array}$ & $\begin{array}{l}\text { Time from HIV diagnosis to viral suppression } \\
(<50 \mathrm{c} / \mathrm{mL}), \text { months }\end{array}$ \\
\hline \multicolumn{4}{|l|}{ Gender } \\
\hline Male & 577 (91.9) & $1.0(0.8,1.4)$ & $9.8(8.1,10.9)$ \\
\hline Female & $51(8.1)$ & $0.6(0.3,1.9)$ & $5.9(4.3,8.3)$ \\
\hline \multicolumn{4}{|l|}{ Place of residence } \\
\hline Zagreb & $273(43.5)$ & $1.2(0.7,3.3)$ & $12.5(10.1,15.1)$ \\
\hline Other than Zagreb & $355(56.5)$ & $1.0(0.7,1.3)$ & $7.7(6.7,9.0)$ \\
\hline \multicolumn{4}{|l|}{ Mode of transmission } \\
\hline MSM & $476(75.8)$ & $1.1(0.8,1.9)$ & $10.1(8.3,11.6)$ \\
\hline IDU & $11(1.7)$ & $3.4(0.1,22.1)$ & $9.9(4.1,35.7)$ \\
\hline Heterosexual contact & $121(19.3)$ & $0.6(0.5,1.0)$ & $6.8(5.8,8.9)$ \\
\hline Unknown & $20(3.2)$ & $0.8(0.2,7.3)$ & $6.5(3.7,21.0)$ \\
\hline \multicolumn{4}{|l|}{ Had AIDS } \\
\hline Yes & $186(29.6)$ & $0.6(0.5,0.7)$ & $6.5(5.8,8.3)$ \\
\hline No & $442(70.4)$ & $2.6(1.3,3.8)$ & $11.1(9.1,13.2)$ \\
\hline \multicolumn{4}{|c|}{ Periods of entry into care, years } \\
\hline $2005-2008$ & $220(35.0)$ & $2.7(1.3,8.6)$ & $11.2(8.6,16.2)$ \\
\hline $2009-2011$ & $176(28.0)$ & $1.0(0.7,2.1)$ & $8.4(6.7,11.6)$ \\
\hline $2012-2014$ & $232(36.9)$ & $0.6(0.5,0.7)$ & $8.1(6.8,10.1)$ \\
\hline \multicolumn{4}{|c|}{ Hepatitis $B$ antigen present at inclusion into care } \\
\hline Yes & $34(5.4)$ & $1.1(0.5,7.3)$ & $7.3(4.2,27.3)$ \\
\hline No & $594(94.6)$ & $1.0(0.7,1.3)$ & $9.7(8.0,10.6)$ \\
\hline \multicolumn{4}{|c|}{ Hepatitis $\mathrm{C}$ antibody positive at inclusion into care } \\
\hline Yes & $19(3.0)$ & $2.4(0.7,13.7)$ & $9.6(4.5,58.5)$ \\
\hline No & $609(97.0)$ & $1.0(0.7,1.3)$ & $9.3(7.9,10.6)$ \\
\hline \multicolumn{4}{|c|}{ CD4 count at inclusion into care cells/ $\mu \mathrm{L}$} \\
\hline$<100$ & 190 & $0.4(0.3,0.5)$ & $6.2(5.8,6.5)$ \\
\hline $100-350$ & 194 & $0.5(0.5,0.7)$ & $6.4(5.9,7.2)$ \\
\hline$\geq 350$ & 244 & $18.5(14.1,23.2)$ & $26.7(21.2,31.1)$ \\
\hline \multicolumn{4}{|l|}{ Gap in care } \\
\hline Yes & 120 & $26.0(8.9,35.5)$ & $38.2(26.9,49.9)$ \\
\hline No & 508 & $0.7(0.6,0.9)$ & $7.5(6.8,8.7)$ \\
\hline \multicolumn{4}{|c|}{ Integration into care, days } \\
\hline $0-7$ & $339(54.0)$ & $0.4(0.3,0.5)$ & $6.5(6.1,7.0)$ \\
\hline $8-29$ & $201(32.0)$ & $3.4(1.2,7.6)$ & $12.1(9.8,15.1)$ \\
\hline$\geq 30$ & $88(14.0)$ & $11.6(3.7,18.5)$ & $19.2(12.3,30.0)$ \\
\hline \multicolumn{4}{|c|}{$\mathrm{VL}>100000$ copies $/ \mathrm{ml}$ at inclusion into care } \\
\hline Yes & $341(54.3)$ & $0.5(0.4,0.7)$ & $7.4(6.6,8.6)$ \\
\hline No & $287(45.7)$ & $8.6(3.8,14.9)$ & $16.1(10.3,21.1)$ \\
\hline
\end{tabular}

Table 2. Time from HIV diagnosis to antiretroviral treatment initiation and to viral suppression $(<50$ copies $/ \mathrm{ml}$ ) in 628 individuals entering care in Croatia from 2005 to 2014 . Values are frequencies, percentages, medians and 95\% confidence intervals (CI). Medians and 95\% CI are derived from the product-limit (Kaplan-Meier) estimates. MSM men who have sex with men, IDU injection drug users, VL, NRTI, nucleoside analogues reverse transcriptase inhibitors, NNRTI nonnucleoside reverse transcriptase inhibitors, $I I$ integrase inhibitors, $P I$ protease inhibitors, $V L$ viral load.

risk (MSM vs not MSM), age and place of residence (Zagreb vs outside of Zagreb) did not have a significant impact on time of ART initiation. MSM had a somewhat longer time to ART initiation than non-MSM but this was significant only at the 60 th and 65 th quantile $(p=0.019$ and 0.014 respectively; Fig. 3$)$. As expected, persons who had a gap in care before ART, initiated ART later compared to those with no gap and this difference was decreasing from lower to higher quantiles. The back-transformation of our log-transformed dependent variable indicated that persons with a gap before ART started ART 10.0 (95\% CI 6.5-15.7) times later compared to those who did not have a gap at the 50th quantile. Calendar year of entry into care (per one year) was significantly associated with earlier ART initiation. At the 50th quantile, an increase of one year resulted in $17.8 \%$ (95\% CI: 13.1-22.2) earlier ART initiation. The effect of calendar year on early ART initiation was less pronounced at lower quantiles.

Higher viral load also resulted in earlier ART initiation. At the 50th quantile an increase of one unit $(\log 10)$ of viral load resulted in 46.5\% (95\% CI 33.2-57.2) earlier ART initiation. Among persons with no clinical AIDS, 


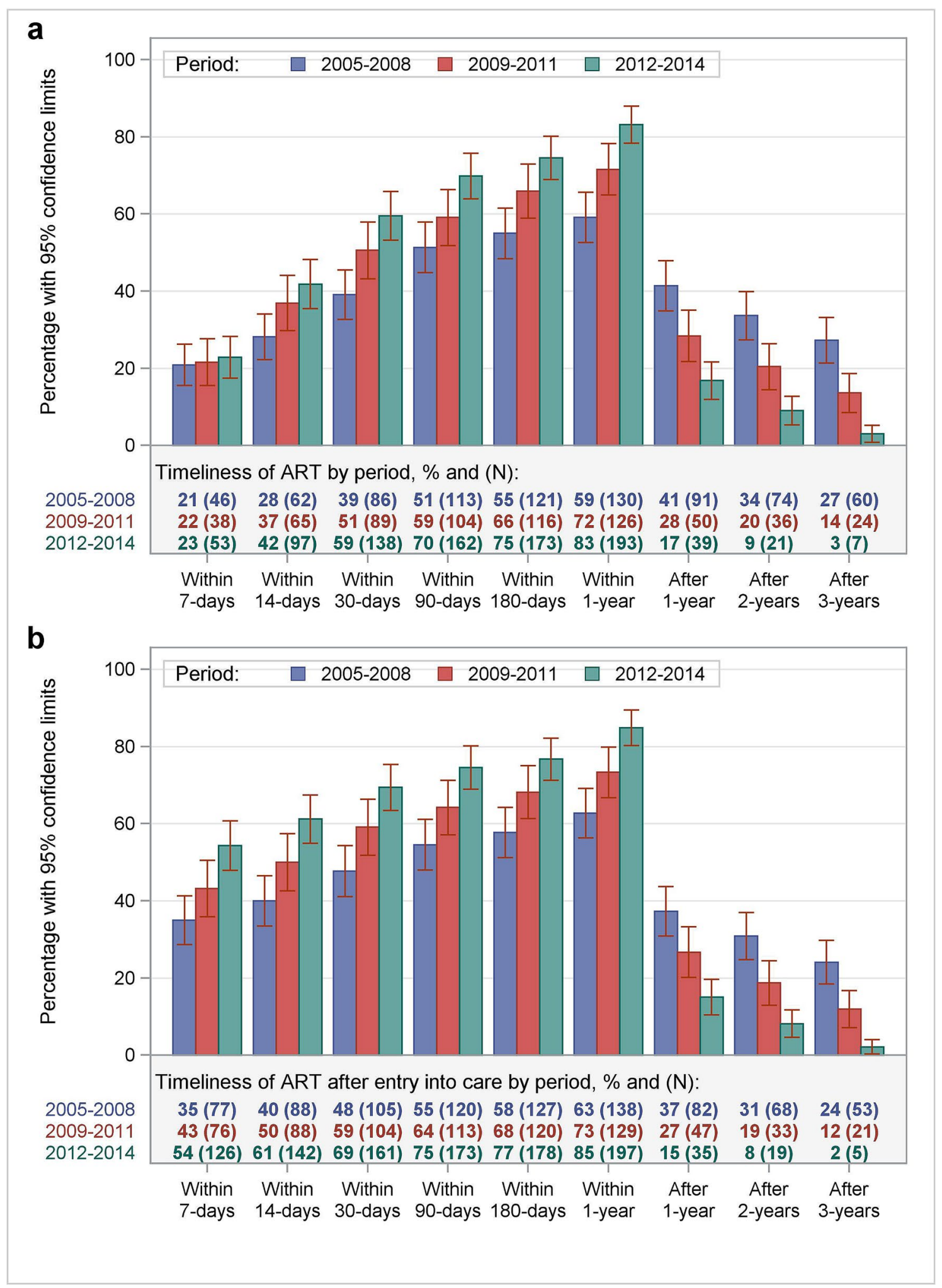

Figure 1. Time from HIV diagnosis to antiretroviral therapy (ART) initiation according to study periods. (a) Shows the frequency distribution of time from HIV diagnosis to ART initiation and (b) shows the frequency distribution of time from entry into care to ART initiation. 


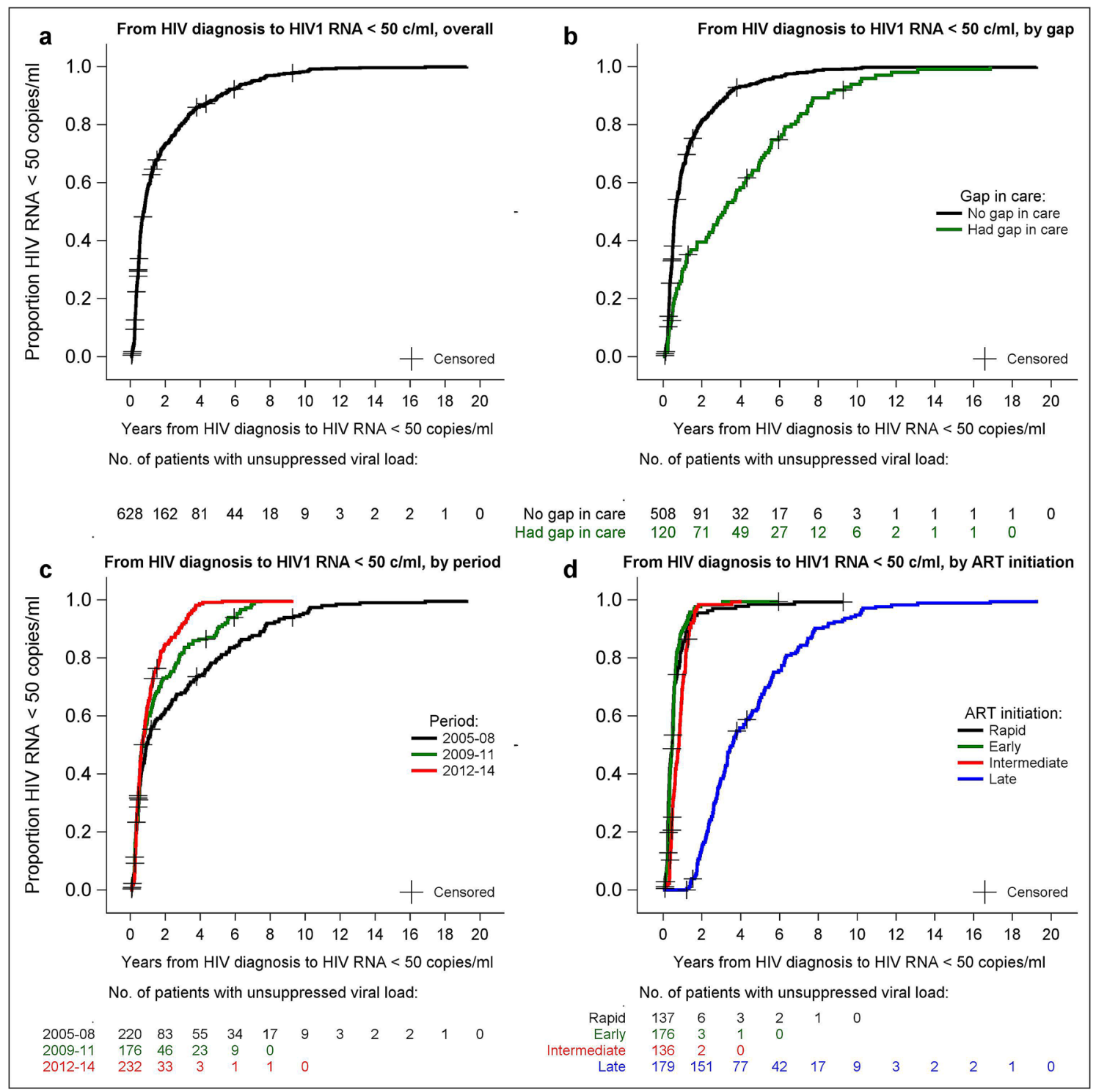

Figure 2. Kaplan-Meier plots of time from HIV diagnosis to undetectable plasma HIV-RNA: (a) overall; (b) according to gap in care; (c) by period of ART initiation; and (d) timeliness of ART initiation (rapid = within 7 days early $=8$ to 30 days, intermediate $=31$ days to one year, late $>1$ year).

started ART 13.3 (95\% CI 8.9-19.9) times later than those with $<200$ CD4 cells per $\mathrm{mm}^{3}$. Also, persons with clinical AIDS started ART later in comparison to persons with CD4 count $<200 / \mathrm{mm}^{3}$, with an increasing difference from low to high quantiles, at the 75th quantile, ART was initiated 2.6 (95\% CI 1.8-3.5) times later. The analysis was also adjusted for the time to integration into care, and as expected integration into care was associated with later ART initiation at all quantiles. The effect of integration into care was more pronounced at lower quantiles.

\section{Discussion}

We report on Croatian national data on time from HIV diagnosis to ART initiation for the period 2005 to 2014. The time from diagnosis to ART initiation decreased by $77.8 \%$ through the study periods and the overall median time from diagnosis to ART initiation was 31 days. The time from HIV diagnosis to first clinical visit also decreased and it was the shortest in 2012-2014 with a median of 7 days. There are very few national estimates on time from HIV diagnosis to ART initiation. In a population-based probability sample of HIV-infected adults receiving medical care in the United States (May 2004-April 2009) the median time to ART initiation was 10 months. A recent report from England available at a preprint server (arXiv:2010.00740) showed that the percentage of MSM on ART within 180 days of diagnosis increased from $34.7 \%$ in 2008 to $84.5 \%$ in 2016 and $91.0 \%$ in 2018. The North American AIDS Cohort Collaboration on Research and Design study group recently reported that the median days from entry into care to ART prescription was 69 days in 2005, 29 days in 2012, and 6 days in 2018. In 2018, 35\% of participants entering HIV care were prescribed ART the same day ${ }^{14}$. Many reports from cohorts, clinic-based or program-based sites have published trends of shorter times to ART initiation in more recent years ${ }^{13,15-22}$ particularly after 2015 when "treat all" policy was introduced by $\mathrm{WHO}^{6}$. 


\begin{tabular}{|c|c|c|c|c|}
\hline & \multicolumn{4}{|c|}{ ART initiation after confirmed HIV diagnosis } \\
\hline & Rapid (within 7 days) & Early 8 to 30 days & Intermediate 31 days- 1 year & Late $>1$ year \\
\hline $\begin{array}{l}\text { Number of patients with }>6 \text { months } \\
\text { follow-up }\end{array}$ & $N=131$ & $\mathrm{~N}=168$ & $N=131$ & $\mathrm{~N}=171$ \\
\hline Virologic failurea, $\%,(\mathrm{n})$ & $22.1(29)$ & $10.1(17)$ & $16.0(21)$ & $14.6(25)$ \\
\hline Resistance tests performed at failure, $n$ & 17 & 10 & 11 & 13 \\
\hline Any resistance at failure, $\mathrm{n}$ & 12 & 4 & 5 & 7 \\
\hline Resuppressed $^{\mathrm{b}}<50$ copies/ml, n/N & $23 / 29$ & $11 / 17$ & $16 / 21$ & $16 / 25$ \\
\hline \multicolumn{5}{|l|}{ Durability of viral load suppression } \\
\hline Among patients who became undetectable & $\mathrm{N}=134$ & $\mathrm{~N}=167$ & $\mathrm{~N}=132$ & $\mathrm{~N}=175$ \\
\hline HIV1-RNA $<50$ copies/ml' $, \%,(\mathrm{n})$ & $92.5(124)$ & $97.0(162)$ & $94.7(125)$ & $92.6(162)$ \\
\hline HIV1-RNA < 200 copies/mlb $\%$, (n) & $94.8(127)$ & $97.6(163)$ & $97.7(129)$ & $94.9(166)$ \\
\hline Timec$^{c}$, median (Q1-Q3), years & $6.2(3.8-9.2)$ & $5.2(3.6-8.0)$ & $6.0(3.9-8.9)$ & $3.9(1.9-6.7)$ \\
\hline All patients & $\mathrm{N}=137$ & $\mathrm{~N}=176$ & $\mathrm{~N}=136$ & $\mathrm{~N}=179$ \\
\hline HIV1-RNA < 50 copies/ml' $, \%,(\mathrm{n})$ & $90.5(124)$ & $92.0(162)$ & $91.9(125)$ & $91.1(163)$ \\
\hline HIV1-RNA < 200 copies/mlb $\%$, (n) & $92.7(127)$ & $93.8(165)$ & $95.6(130)$ & $93.3(167)$ \\
\hline Timec$^{\mathrm{c}}$, median (Q1-Q3), years & $6.0(3.8-9.1)$ & $5.1(3.5-7.8)$ & $5.9(3.8-8.7)$ & $3.9(1.9-6.6)$ \\
\hline
\end{tabular}

Table 3. Number of patients with viral load suppression, failure, resistance and durability of viral suppression according to timeliness of ART initiation, 2005-2014. ART antiretroviral therapy. ${ }^{\text {a }} \mathrm{A}$ single viral load measurement of HIV1-RNA $>1000$ copies/ml at any time after 6 months of ART initiation (including patients who stopped ART). ${ }^{\text {b}}$ Viral load at last measurement. ${ }^{c}$ Time from antiretroviral therapy initiation to last HIV viral load measurement.

Our data indicate that immediate ART provision in Croatia seemed to predate the WHO guideline changes made in 2015. ART initiation within 30 days of HIV diagnosis was already present in almost $30 \%$ of persons entering care with a CD4 cell count $>350$ per $\mathrm{mm}^{3}$ in the period 2012-2014. There is a paucity of data on the durability of viral suppression in early ART starters, our data indicate that early ART initiation is associated with durable viral suppression over a median of 5-year follow-up. Overall, 15\% of patients had at least one measurement of HIV-1 RNA > 1000 copies/ml after 6-months of ART, and this figure was highest among rapid ART initiators (29/131, 22\%, Table 3). Also, numerically, rapid starters had more HIV-1 resistance at failure (Table 3). However, this should be interpreted with caution as the number of patients in our study was relatively small. It is encouraging that the majority of persons who experienced virological failure where latter resuppressed (Table 3). Nevertheless, more studies on the durability, virological failure and resistance after rapid and early ART initiation are needed.

Differences in the timing of ART initiation among various patient categories may be explained by different factors. For example, a preference for beginning drug therapy as soon as possible for patients with difficulty reaching the treatment center may explain the difference in care among patients who live outside the city versus in Zagreb. Introduction of ART also depended on the severity of HIV infection and thus patients with lower CD4 cell counts and higher viral RNA load initiated ART earlier. Patients with advanced age may have been deemed at risk for poor health outcomes due to HIV and were initiated with ART sooner than younger (possibly deemed healthier) counterparts. Heterosexual mode of transmission is associated with more advanced HIV disease at diagnosis in Croatia ${ }^{23}$, so the longer time to ART initiation in MSM seen on bivariable analysis was largely attenuated in multivariable analysis. However, other factors such as for example fear of side effects might be more pronounced in MSM comparted to heterosexuals which then might have resulted in a delay in ART initiation.

The overall linkage to care in our study was within 7 days from HIV diagnosis in $54.0 \%$ and within 30 days in $86.0 \%$, which is higher than reported in some other studies ${ }^{20,24,25}$. Still, the question on how quickly ART should be initiated after the confirmed or even suspected HIV diagnosis has been of major focus in recent years. Several randomized trials showed that rapid ART (including the same day of diagnosis) could improve patient outcomes by reducing loss to care and more rapidly achieving an undetectable viral load and thus reducing the possibility of onward HIV transmission ${ }^{26-33}$. Rapid ART initiation could also decrease health costs ${ }^{34}$. On the other hand, a study on the Dat'AIDS cohort showed that a longer period of time between first clinical visit and ART initiation was associated with a better 1-year retention in care ${ }^{13}$. In our study, the gap in care after early ART initiation was as frequent as in those starting ART later.

We used quantile regression in our analysis which is not frequently used to describe associations. The results of quantile regression are interpreted in a similar way to ordinary least squares regression, however unlike predicting the mean, quantile regression is performed at different quantiles of the dependent variable. So, this method provides a more detailed analysis of associations between variables and could be used in studies examining the timeliness of ART initiation.

The inability to establish causation is a limitation of our observational study. For example, while a delay in ART initiation might cause a gap in care, this delay might also be just a consequence of the gap caused by some other factor. Nevertheless, it is encouraging that early ART initiation, without extensive counseling did not result in worse retention in care and did result in achieving a more rapid viral load suppression. While early ART initiation was initially motivated by having a subsequent visit for patients not living in Zagreb, it has been 


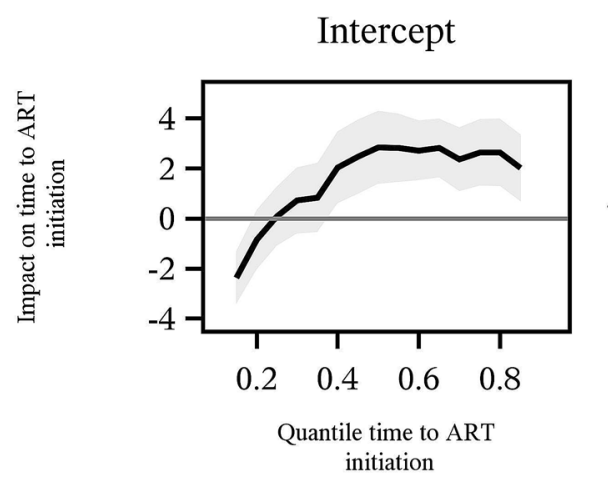

Calendar year, per 1 year

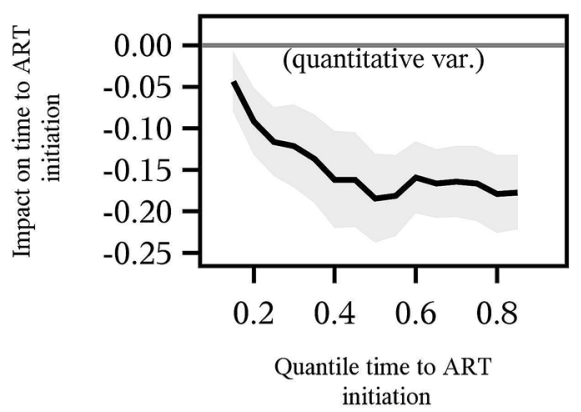

Viral load, per $1 \log 10$

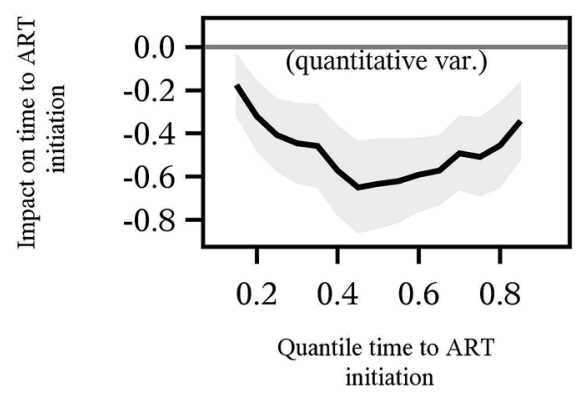

Age, per 10 years

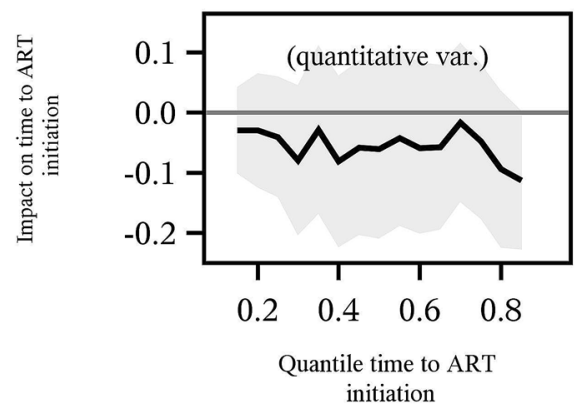

Had gap before ART

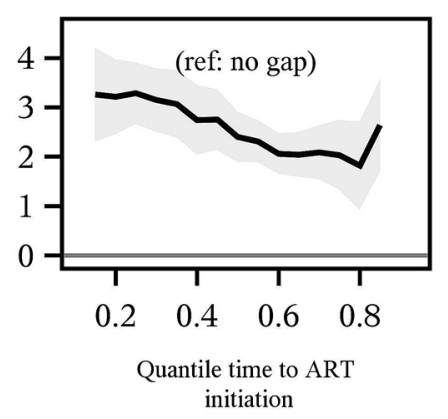

Gender

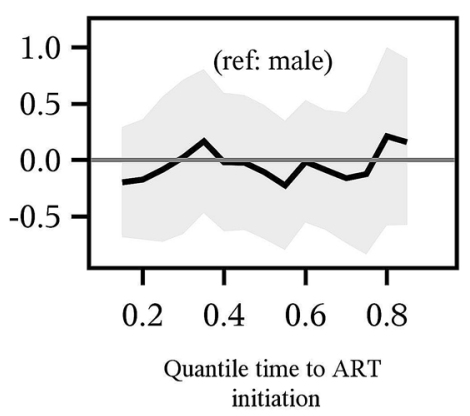

CD4 cell count $\geq 200 / \mathrm{mm}^{3}$

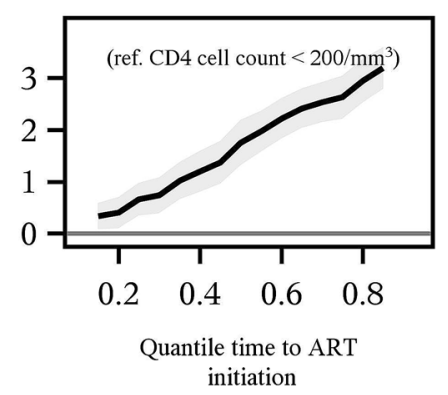

Living in Zagreb

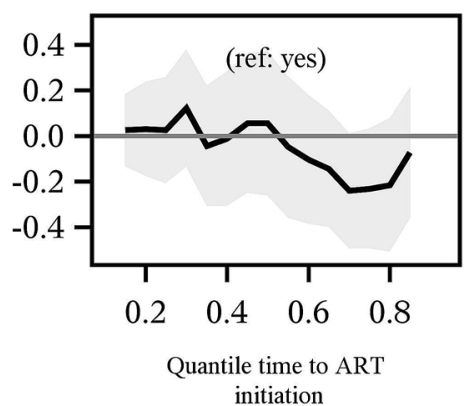

Had gap after ART

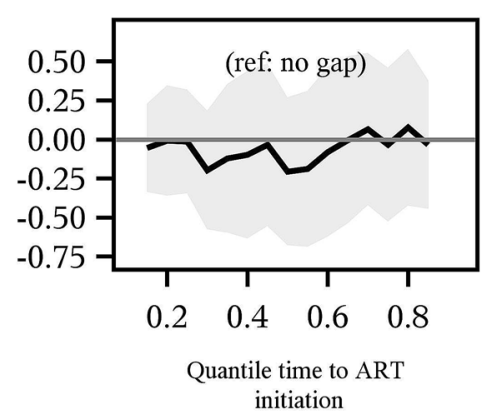

Transmission risk

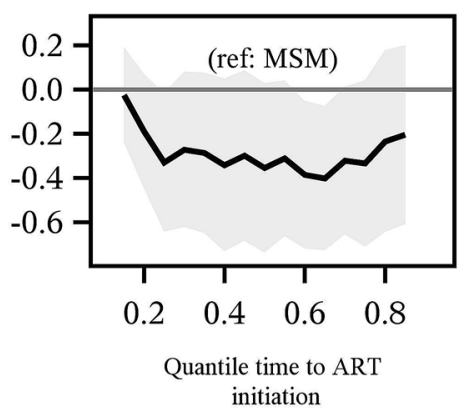

Clinical AIDS

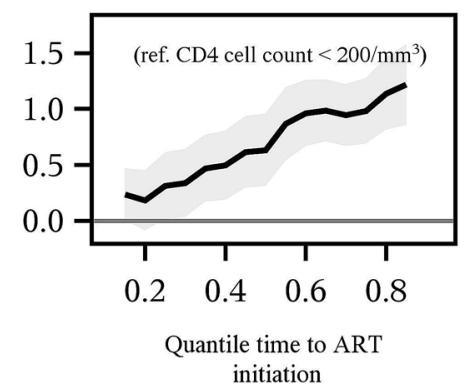

Integration into care

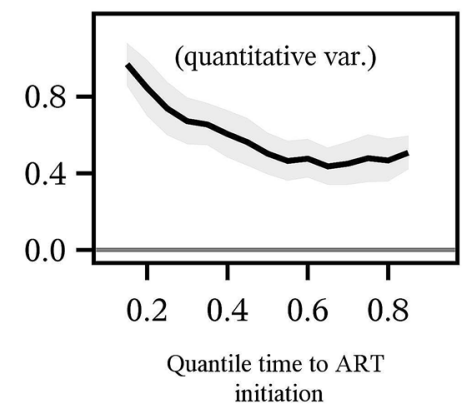

Figure 3. Multivariable quantile regression exploring time from ART initiation and potential related factors. The $\mathrm{x}$-axis represents quantiles of the distribution of the time to ART initiation (log-time in months), and the $y$-axis represents the parameter estimates (coefficients) change in time associated with a one-unit change of the studied covariate, holding other covariates constant. This change is considered significant at a particular quantile when the associated 95\% confidence interval (shaded area) does not cross the 0 line. Integration into care was modeled as log-days. 
slowly adopted for all patients. Another concern is measuring time to viral suppression which is dependent on the frequency of viral load measurement, so the true time of becoming undetectable is unknown. For example, in our study the time to ART initiation decreased over time by $77.8 \%$ whereas the time to reaching an undetectable viral load decreased by $27.7 \%$. We presented our data on durability of VL by the date of last measurement and not by a certain number of years after ART initiation. This has been done because patients might have regular ART refills at the hospital pharmacy and no viral load measurement. However, since the median time of last viral load measurement from ART initiation was quite long for rapid ART starters ( $>6.0$ years), we believe that we did provide data suggesting durability of VL suppression in those who start ART rapidly. Virologic failure was defined by a single plasma HIV-1 RNA $>1000$ copies/ml which is more stringent that the current WHO definition $^{7}$ which requires two measurements. Confirmation was not required for the definition of virologic failure because of less frequent viral load monitoring, and this is also why did not use other definitions of virological failure (for example confirmed $>50$ or $>200 \mathrm{HIV}-1$ RNA copies $/ \mathrm{ml}$ ). Nevertheless, as the majority of patients who failed were resuppressed to $<50$ copies $/ \mathrm{ml}$ the choice of virologic failure cut-off seems not to be of critical importance for our analysis.

In conclusion, our study adds to the growing data of feasibility and beneficence of early initiation of ART and questions the need of extensive counseling prior to ART initiation. Our data support current HIV care guidelines emphasizing early ART initiation. Earlier initiation of ART was not associated with a more frequent subsequent gap in care and our findings also suggest that early ART initiation may have favorable long term virologic outcomes.

\section{Methods}

Setting. Croatia has a centralized system of HIV care, and all Croatian citizens have universal health insurance. All PLWH are treated at the University Hospital for Infectious Diseases (UHID) in Zagreb where they receive regular clinical medical evaluations, including measurements of CD4 cell counts and HIV-RNA viral $\operatorname{load}^{35,36}$. HIV resistance testing is also available but not regularly performed unless in the event of drug failure. The UHID pharmacy dispenses ART drugs for all PLWH and clinicians verify that the patient has the drugs before he or she leaves the hospital. Since 1997, patient information has been kept in an electronic database which catalogues demographic data, dates of clinic visits, prescriptions of ART drugs, CD4 counts, viral load measurements, and other biochemistry tests.

For patients living outside of Zagreb, the centralized care system means that they must travel to the city for receiving HIV care and treatment and for many the journey may be lengthy or difficult. This led UHID physicians to adopt a policy of reducing appointment frequency when feasible ${ }^{35}$. This means that many patients at the UHID receive ART prescriptions for longer periods of time, usually six months. In a typical case, patients begin ART with a month-long prescription, then the next prescription is for two months and then for three. Three-month long ART prescriptions last until the physician is comfortable with providing the patient ART drugs for six months ${ }^{35}$. This is common when the HIV infection is well controlled and the patient requires clinical examination once or twice per year.

Study population and definitions. We utilized anonymized data from the electronic UHID database. The cohort was comprised of Croatian citizens or permanent residents at least 18 years of age who arrived to the UHID for HIV treatment between 2005 and 2014 and had not yet received HIV care elsewhere. Pregnant women were excluded from the study. A total of 628 persons who initiated ART were included in the study cohort. The flow diagram for selecting the study cohort is shown in Fig. 4.

The date of HIV diagnosis was defined as the date when blood was drawn for confirmatory testing. The date of a patient's first clinical visit was considered the starting date of care when a CD4 cell count and viral load was measured. The follow-up ended on December 31, 2017. Patients were censored at the date of their death, relocation from Croatia, or their LTFU. They were considered to have a gap in care if more than one year passed without follow-up. Patients with a gap in care were also considered LTFU if they were alive and living in Croatia and had no outpatient visit for more than 12 months without returning by December 31, 2017.

The study was approved by the Ethics Committee of the University Hospital for Infectious Diseases (UHID), Zagreb, Croatia. The study was performed in accordance with the Declaration of Helsinki and Code of Medical Ethics and Deontology of the Croatian Medical Chamber. Written informed consents was obtained from all participants included in this study.

Viral load and resistance. HIV viral load was determined by various methods: the Cobas AmpliPrep Cobas Amlicor HIV-1 Monitor Test, Version 1 (lower detection limit 200 copies/ml), the ultrasensitive Amplicor HIV-1 Monitor test (lower detection limit 50 copies/ml), the Cobas Ampliprep/Cobas Taqman HIV-1 test, v2.0 (detection limit 20 copies/ml), all from Roche Molecular Systems, Branchburg, NJ and the Abbott RealTime HIV-1 Viral Load assay (detection limit 40 copies/ml). The Cobas AmpliPrep Cobas Amplicor HIV-1 Monitor Test, Version 1 and the ultrasensitive Amplicor HIV-1 Monitor test were used till the end of 2015. The Cobas Ampliprep/Cobas Taqman HIV-1 test was used from September 2009 till the end of study (December 2017) and the Abbott RealTime HIV-1 Viral Load assay from November 2011 till end of study.

HIV resistance testing was done with HIV-1 sequencing using the TRUGENE HIV-1 Genotyping Kit (Visible Genetics, Toronto, Canada) or by Sanger sequencing as previously described ${ }^{37,38}$. Virologic failure was defined as a single HIV-1 RNA of $>1000$ copies/ml at any time after 6 months of ART. Clinically relevant resistance to NRTIs, NNRTIs or PIs was evaluated with Stanford University HIV Drug Resistance Database, Genotypic Resistance Interpretation Algorithm and IAS Drug Resistance Mutation list. A patient was considered to have virological failure with resistance if he harbored at least one mutation from the abovementioned algorithms. 


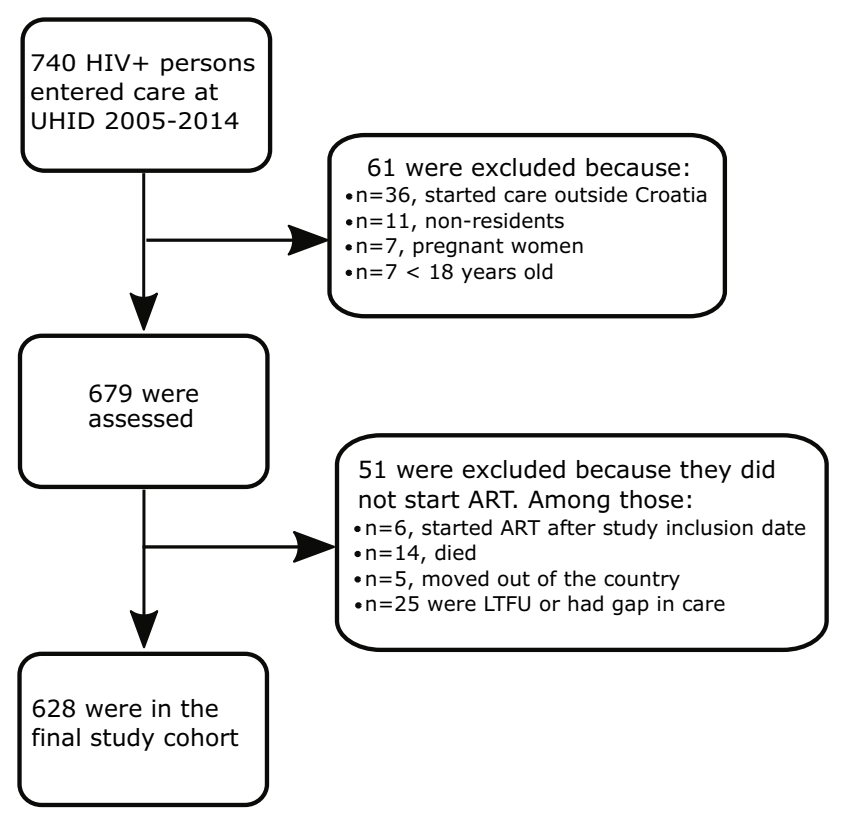

Figure 4. Flow diagram of participants included into the study. HIV human immunodeficiency virus; UHID University Hospital for Infectious Diseases, Zagreb, Croatia; ART antiretroviral treatment; LTFU lost to follow-up.

Statistical methods. The time from HIV diagnosis to ART initiation was categorized: within 7 days ("rapid"), from 8 to 30 days ("early"), from 31 days to one year ("intermediate"), and more than one year ("late"). We assessed how ART initiation timing periods were related to the following: gap in care, gender, transmission mode, CD4 count (cells per $\mu \mathrm{L}$ ), HIV-RNA viral load (categorized as greater or less than $10^{5}$ copies $/ \mathrm{mL}$ ), residence in or outside of Zagreb, whether or not clinical AIDS was present, presence of hepatitis B antigen or hepatitis $\mathrm{C}$ antibody positivity at inclusion into care, initial ART type, and year of entry into care. We also assessed the durability of viral load suppression by the proportion of persons with plasma HIV1-RNA $<50$ and $<200$ copies/ $\mathrm{ml}$ at last follow-up measurement.

We present our data on the study population by frequencies and percentages or median with first and third quartiles (Q1-Q3). Trends in the timeliness of ART initiation were examined by the Cochran-Armitage test. The Jonckheere-Terpstra test was used for comparison of doubly ordered variables, and the Wilcoxon-Mann-Whitney test was used for comparison of groups with continuous or ordinal variables. The incidence of gap in care before and after ART initiation was assessed by the Poisson method. Kaplan-Meier estimators were used to measure time from HIV diagnosis to antiretroviral treatment initiation and to viral suppression defined as a viral load $<50$ copies $/ \mathrm{mL}$. Patients were censored if death $(n=15)$ or lost to follow-up $(n=5)$ occurred before reaching an undetectable viral load.

We also conducted quantile regression analysis to study factors related to ART initiation with gap in care considered the major independent (predictor) variable. Gap in care was categorized as no gap, gap before ART, and gap after ART start. Persons with both gap before and after ART start were excluded ( $\mathrm{n}=9)$, so this analysis included 619 participants. The time from HIV diagnosis to ART initiation was the outcome variable (log months). Age, viral load $(\log 10)$ calendar year and time to integration into care (log days) were modeled as continuous predictor variables. There were concerns about collinearity between the CD4 cell count and clinical AIDS, so we categorized those variables into three categories: (1) CD 4 cell count $<200$ per $\mathrm{mm}^{3}$ and no clinical AIDS, (2) clinical AIDS regardless of CD4 count and (3) CD4 cell count $\geq 200$ per $\mathrm{mm}^{3}$ and no clinical AIDS. Other variables included into the model were gender, residence (living in Zagreb vs outside of Zagreb), and transmission risk (MSM vs not MSM).

Quantile regression has the advantage to provide a more exact description of the distribution of the determinants of time to ART initiation at different quantile (percentile) levels. The coefficients were estimated for each 5 percentiles from 5 to 95 th quantile of time to ART initiation. Standard errors and $95 \%$ confidence intervals (CI) were estimated using resampling with 1000 repetitions. The coefficients for each quantile were plotted as well as their 95\% CI. We used SAS software system release 9.4 (SAS Institute, Cary, NC) to perform the analyses. Two-sided P-values $<0.05$ were considered significant.

Received: 2 August 2020; Accepted: 6 May 2021

Published online: 18 May 2021 


\section{References}

1. World Health Organization. Antiretroviral Therapy for HIV Infection in Adults and Adolescents: Recommendations for a Public Health Approach. 2010 Revision. http://whqlibdoc.who.int/publications/2010/9789241599764_eng.pdf. Accessed 3 August 2010.

2. Group, I. S. S. et al. Initiation of antiretroviral therapy in early asymptomatic HIV infection. N. Engl. J. Med. 373, 795-807. https:// doi.org/10.1056/NEJMoa1506816 (2015).

3. Group, T. A. S. et al. A trial of early antiretrovirals and isoniazid preventive therapy in Africa. N. Engl. J. Med. 373, 808-822. https:// doi.org/10.1056/NEJMoa1507198 (2015).

4. Kitahata, M. M. et al. Effect of early versus deferred antiretroviral therapy for HIV on survival. N. Engl. J. Med. 360, 1815-1826. https://doi.org/10.1056/NEJMoa0807252 (2009).

5. Lodi, S. et al. Comparative effectiveness of immediate antiretroviral therapy versus CD4-based initiation in HIV-positive individuals in high-income countries: Observational cohort study. Lancet HIV 2, e335-343. https://doi.org/10.1016/S2352-3018(15)00108-3 (2015).

6. World Health Organization. Guideline on When to Start Antiretroviral Therapy and on Pre-exposure Prophylaxis for HIV. www. who.int/hiv/pub/guidelines/earlyrelease-arv/en/ (accessed July 2020) (2015).

7. World Health Organization. Guidelines for managing advanced HIV disease and rapid initiation of antiretroviral therapy, July 2017. Geneva: World Health Organization; 2017. Licence: CC BY-NC-SA 3.0 IGO.

8. European AIDS Clinical Society (EACS). Guidelines for the clinical Management and Treatment of HIV-Infected Adults. Version 10.1. October 2020. https://www.eacsociety.org/guidelines/eacs-guidelines/eacs-guidelines.html.

9. Department of Health and Human Services (DHHS). Guidelines for the Use of Antiretroviral Agents in HIV-1-Infected Adults and Adolescents December 18, 2019. https://www.AIDSinfonihgov. (Accessed July 2020).

10. Boyd, M. A., Boffito, M., Castagna, A. \& Estrada, V. Rapid initiation of antiretroviral therapy at HIV diagnosis: Definition, process, knowledge gaps. HIV Med 20(Suppl 1), 3-11. https://doi.org/10.1111/hiv.12708 (2019).

11. Coffey, S. et al. RAPID antiretroviral therapy: High virologic suppression rates with immediate antiretroviral therapy initiation in a vulnerable urban clinic population. AIDS 33, 825-832. https://doi.org/10.1097/QAD.0000000000002124 (2019).

12. Robertson, M. M., Braunstein, S. L., Hoover, D. R., Li, S. \& Nash, D. Timeliness of human immunodeficiency virus diagnosis and antiretroviral treatment initiation in the era of universal testing and treatment. J. Infect. Dis. 220, 648-656. https://doi.org/10.1093/ infdis/jiz148 (2019).

13. Cuzin, L. et al. Too fast to stay on track? Shorter time to first anti-retroviral regimen is not associated with better retention in care in the French Dat'AIDS cohort. PLoS ONE 14, e0222067. https://doi.org/10.1371/journal.pone.0222067 (2019).

14. Lee, J. S. et al. CD4 count at entry into HIV care and at antiretroviral therapy prescription in the US, 2005-2018. Clin. Infect. Dis. https://doi.org/10.1093/cid/ciaa1904 (2020).

15. Le Guillou, A. et al. Reaching the Second and Third Joint United Nations Programme on HIV/AIDS 90-90-90 targets is accompanied by a dramatic reduction in primary human immunodeficiency virus (HIV) infection and in recent HIV infections in a large French nationwide HIV cohort. Clin. Infect. Dis. 71, 293-300. https://doi.org/10.1093/cid/ciz800 (2020).

16. Medland, N. A. et al. Time from HIV diagnosis to commencement of antiretroviral therapy as an indicator to supplement the HIV cascade: Dramatic fall from 2011 to 2015. PLoS ONE 12, e0177634. https://doi.org/10.1371/journal.pone.0177634 (2017).

17. Sloan, D. J. et al. Evidence of improving antiretroviral therapy treatment delays: An analysis of eight years of programmatic outcomes in Blantyre, Malawi. BMC Public Health 13, 490. https://doi.org/10.1186/1471-2458-13-490 (2013).

18. Whitlock, G., Carbonell, M., Blackwell, S., Nwokolo, N. \& Dean Street Collaborative, G. Rapid initiation of antiretroviral therapy in those with newly diagnosed HIV infection in London, UK. HIV Med. 20, 699-703. https://doi.org/10.1111/hiv.12780 (2019).

19. Huang, Y. C. et al. Short-term outcomes of rapid initiation of antiretroviral therapy among HIV-positive patients: real-world experience from a single-centre retrospective cohort in Taiwan. BMJ Open 9, e033246. https://doi.org/10.1136/bmjopen-2019-033246 (2019).

20. Linn, K. Z. et al. Time to anti-retroviral therapy among people living with HIV enrolled into care in Myanmar: How prepared are we for 'test and treat'?. Glob. Health Action 11, 1520473. https://doi.org/10.1080/16549716.2018.1520473 (2018).

21. Robertson, M. M., Braunstein, S. L., Hoover, D. R., Li, S. \& Nash, D. Timeliness of HIV diagnosis and antiretroviral treatment initiation in the era of universal test and treat. J. Infect. Dis. https://doi.org/10.1093/infdis/jiz148 (2019).

22. Tymejczyk, O. et al. Impact of universal antiretroviral treatment eligibility on rapid treatment initiation among young adolescents with human immunodeficiency virus in Sub-Saharan Africa. J. Infect. Dis.. 222, 755-764. https://doi.org/10.1093/infdis/jiz547 (2020).

23. Begovac, J., Gedike, K., Lukas, D. \& Lepej, S. Z. Late presentation to care for HIV infection in Croatia and the effect of interventions during the Croatian Global Fund Project. AIDS Behav. 12, S48-53. https://doi.org/10.1007/s10461-008-9398-9 (2008).

24. Zhao, Y. et al. Immediate antiretroviral therapy decreases mortality among patients with high CD4 counts in China: A nationwide retrospective cohort study. Clin. Infect. Dis. 66, 727-734. https://doi.org/10.1093/cid/cix878 (2018).

25. Wilton, J. et al. Trends in HIV care cascade engagement among diagnosed people living with HIV in Ontario, Canada: A retrospective, population-based cohort study. PLoS ONE 14, e0210096. https://doi.org/10.1371/journal.pone.0210096 (2019).

26. Amanyire, G. et al. Effects of a multicomponent intervention to streamline initiation of antiretroviral therapy in Africa: A steppedwedge cluster-randomised trial. Lancet HIV 3, e539-e548. https://doi.org/10.1016/S2352-3018(16)30090-X (2016).

27. Labhardt, N. D. et al. Effect of offering same-day ART vs usual health facility referral during home-based HIV testing on linkage to care and viral suppression among adults with HIV in Lesotho: The CASCADE randomized clinical trial. JAMA 319, 1103-1112. https://doi.org/10.1001/jama.2018.1818 (2018).

28. Rosen, S. et al. Initiating antiretroviral therapy for HIV at a patient's first clinic visit: The RapIT randomized controlled trial. PLoS Med. 13, e1002015. https://doi.org/10.1371/journal.pmed.1002015 (2016).

29. Koenig, S. P. et al. Same-day HIV testing with initiation of antiretroviral therapy versus standard care for persons living with HIV: A randomized unblinded trial. PLoS Med 14, e1002357. https://doi.org/10.1371/journal.pmed.1002357 (2017).

30. Mateo-Urdiales, A., Johnson, S., Smith, R., Nachega, J. B. \& Eshun-Wilson, I. Rapid initiation of antiretroviral therapy for people living with HIV. Cochrane Database Syst. Rev. 6, CD012962. https://doi.org/10.1002/14651858.CD012962.pub2 (2019).

31. Elul, B. et al. A combination intervention strategy to improve linkage to and retention in HIV care following diagnosis in Mozambique: A cluster-randomized study. PLoS Med. 14, e1002433. https://doi.org/10.1371/journal.pmed.1002433 (2017).

32. McNairy, M. L. et al. Effectiveness of a combination strategy for linkage and retention in adult HIV care in Swaziland: The Link4Health cluster randomized trial. PLoS Med. 14, e1002420. https://doi.org/10.1371/journal.pmed.1002420 (2017).

33. Stevens, W. S. et al. Multidisciplinary point-of-care testing in south African primary health care clinics accelerates HIV ART initiation but does not alter retention in care. J. Acquir. Immune Defic. Syndr. 76, 65-73. https://doi.org/10.1097/QAI.0000000000 001456 (2017).

34. Benson, C. et al. Rapid initiation of antiretroviral therapy following diagnosis of human immunodeficiency virus among patients with commercial insurance coverage. J. Manag. Care Spec. Pharm. 26, 129-141. https://doi.org/10.18553/jmcp.2019.19175 (2020).

35. Romih, V., Zidovec Lepej, S., Gedike, K., Lukas, D. \& Begovac, J. Frequency of HIV-1 viral load monitoring of patients initially successfully treated with combination antiretroviral therapy. PLOS ONE 5, e15051. https://doi.org/10.1371/journal.pone.0015051 (2010). 
36. Begovac, J., Zekan, A. \& Skoko-Poljak, D. Twenty years of human immunodeficiency virus infection in Croatia: An epidemic that is still in an early stage. Coll. Antropol. 30(Suppl 2), 17-23 (2006).

37. Grgic, I. et al. The prevalence of transmitted drug resistance in newly diagnosed HIV-infected individuals in Croatia: The role of transmission clusters of men who have sex with men carrying the T215S surveillance drug resistance mutation. AIDS Res. Hum. Retrovir. 29, 329-336. https://doi.org/10.1089/AID.2012.0191 (2013).

38. Oroz, M. et al. Analysis of HIV-1 diversity, primary drug resistance and transmission networks in Croatia. Sci. Rep. 9, 17307. https://doi.org/10.1038/s41598-019-53520-8 (2019).

\section{Acknowledgements}

This study has been partially reported in the 2020 Abstract Book of the 30th ECCMID 2020 conference Abstract No. 2879.

\section{Author contributions}

N.B. participated in study design, in writing and revision of manuscript; L.B. participated in literature review, writing and revisions of the manuscript. D.L. and S.Z. participated in data collection, writing and critical review of manuscript. J.B. participated in study design, data analysis, figure preparation, writing and revision of manuscript.

\section{Funding}

This study was supported by the Croatian Science Foundation (project no. IP-2014-09-4461 and IP-2019-049702) principal investigator: Josip Begovac.

\section{Competing interests}

The authors declare no competing interests.

\section{Additional information}

Correspondence and requests for materials should be addressed to J.B.

Reprints and permissions information is available at www.nature.com/reprints.

Publisher's note Springer Nature remains neutral with regard to jurisdictional claims in published maps and institutional affiliations.

Open Access This article is licensed under a Creative Commons Attribution 4.0 International License, which permits use, sharing, adaptation, distribution and reproduction in any medium or format, as long as you give appropriate credit to the original author(s) and the source, provide a link to the Creative Commons licence, and indicate if changes were made. The images or other third party material in this article are included in the article's Creative Commons licence, unless indicated otherwise in a credit line to the material. If material is not included in the article's Creative Commons licence and your intended use is not permitted by statutory regulation or exceeds the permitted use, you will need to obtain permission directly from the copyright holder. To view a copy of this licence, visit http://creativecommons.org/licenses/by/4.0/.

(c) The Author(s) 2021 